\title{
Organelle membrane-specific chemical labeling and dynamic imaging in living cells
}

\section{$\operatorname{AUTHOR}(\mathrm{S}):$}

Tamura, Tomonori; Fujisawa, Alma; Tsuchiya, Masaki; Shen, Yuying; Nagao, Kohjiro; Kawano, Shin; Tamura, Yasushi; Endo, Toshiya; Umeda, Masato; Hamachi, Itaru

\section{CITATION:}

Tamura, Tomonori ... [et al]. Organelle membrane-specific chemical labeling and dynamic imaging in living cells. Nature Chemical Biology 2020, 16(12): 1361-1367

\section{ISSUE DATE:}

2020-12

URL:

http://hdl.handle.net/2433/259230

\section{RIGHT:}

This is the accepted manuscript of the article, which has been published in final form at https://doi.org/10.1038/s41589020-00651-z:; The full-text file will be made open to the public on 21 March 2021 in accordance with publisher's 'Terms and Conditions for Self-Archiving'.; この論文は出版社版でありません。引用の際には出版社版をご確認ご利用ください 。; This is not the published version. Please cite only the published version. 


\section{Organelle membrane-specific chemical labelling and dynamic imaging in living cells}

Tomonori Tamura, ${ }^{1,2}$ Alma Fujisawa, ${ }^{1,2}$ Masaki Tsuchiya, ${ }^{1,2}$ Yuying Shen, ${ }^{1}$ Kohjiro Nagao, ${ }^{1}$ Shin Kawano, ${ }^{3,4}$ Yasushi Tamura, ${ }^{5}$ Toshiya Endo, ${ }^{3,4}$ Masato Umeda ${ }^{1} \&$ Itaru Hamachi ${ }^{1,2^{*}}$

${ }^{1}$ Department of Synthetic Chemistry and Biological Chemistry, Graduate School of Engineering, Kyoto University, Katsura, Nishikyo-ku, Kyoto 615-8510, Japan

${ }^{2}$ JST-ERATO, Hamachi Innovative Molecular Technology for Neuroscience, Kyoto, Japan

${ }^{3}$ Faculty of Life Sciences, Kyoto Sangyo University, Kyoto 603-8555, Japan.

${ }^{4}$ Institute for Protein Dynamics, Kyoto Sangyo University, Kyoto 603-8555, Japan.

${ }^{5}$ Faculty of Science, Yamagata University, Yamagata 990-8560, Japan

Correspondence should be addressed to I.H. (ihamachi@sbchem.kyoto-u.ac.jp). 


\begin{abstract}
Lipids play crucial roles as the structural elements, signalling molecules, and material transporters in cells. However, the functions and dynamics of lipids within cells remain unclear because of a lack of methods to selectively label lipids in specific organelles and trace their movement by live-cell imaging. We describe here a technology for the selective labelling and fluorescence micro/nanoscopic imaging of phosphatidylcholines in target organelles. The approach involves the metabolic incorporation of azido-choline followed by a spatially limited bioorthogonal reaction, which enables the visualization and quantitative analysis of interorganelle lipid transport in live cells. Most importantly, with live-cell imaging, we obtained direct evidence that the autophagosome membrane originates from the endoplasmic reticulum. This method is simple and robust, and thus powerful for real-time tracing of interorganelle lipid trafficking.
\end{abstract}

\title{
Introduction
}

In eukaryotic cells, biological membranes not only provide a physical interface between cell interiors and their environment, but also define the structure and function of intracellular organelles. Choline-containing phospholipids (CPLs), including phosphatidylcholine (PC) and sphingomyelin (SM), are the major components of eukaryotic cell membranes and play critical roles in structural integrity of the membrane bilayer, regulation of cell signalling and membrane protein functions. ${ }^{1}$ Therefore, investigation of the intracellular behaviour of CPLs is essential for deep understanding of many physiological processes. CPLs are mainly synthesized in the endoplasmic reticulum (ER) and/or Golgi apparatus, organelles of the eukaryotic cell, and subsequently translocate to other organelles through vesicular transport, monomeric diffusion mediated by lipid transfer proteins, or direct membrane contact. ${ }^{2}$ Although such interorganelle lipid transport is a fundamental mechanism for maintaining cellular membrane systems, subcellular dynamics of CPLs are poorly understood, primarily because of a lack of methods that allow imaging of CPLs in specific organelles of live cells. ${ }^{3-5}$ Pulse-labelling of CPLs newly biosynthesized in the ER with $\left[{ }^{3} \mathrm{H}\right]$-choline has been previously used to study the transport of CPLs from the ER to the plasma membrane (PM) ${ }^{6}$ However, this approach does not 
allow real-time tracking of CPLs. In addition, this requires rapid isolation of organelle membranes, which often suffers from contamination of other organelles and thereby compromises data interpretation. Fluorescent lipid analogues and lipid-binding protein (e.g. lysenin)-fluorophore conjugates are widely utilized to visualize CPLs in live cells but do not have organelle selectivity in general. ${ }^{3-5}$ More recently, some organelle membranes have been visualized by high density environmentally sensitive (HIDE) probes consisting a trans-cyclooctene (TCO)-tethered organelle-specific lipid (or lipid-like small molecule) that is linked with silicon-rhodamine dye in cells. ${ }^{7,8}$ Indeed this method is useful in super resolution imaging of organelle membranes, but it does not allow the visualization of CPLs because there are no TCO-modified CPLs that can localize in a specific organelle.

Here, we report a chemical strategy for selective labelling and fluorescence micro/nanoscopic imaging of CPLs in ER/Golgi or mitochondria. This relies on the metabolic incorporation of azido-choline $\left(\mathrm{N}_{3}-\mathrm{Cho}\right)$ into CPLs of whole membranes of cells, ${ }^{9}$ followed by spatially limited strain-promoted alkyne-azide cycloaddition (SPAAC) with organelle-localizable click reagents (OCRs) bearing a strained alkyne (Figure 1a). ${ }^{10,11}$ In this study, we developed OCRs for the ER/Golgi and mitochondria, by which specific labelling of azide-tethered PCs residing in the target organelles of several types of cells was achieved. This method allows for time-lapse imaging and quantitative analysis of the translocation of PCs from ER/Golgi- or mitochondrial membranes to other organelles. Furthermore, we used it to show clearly that autophagosomal membranes originate from ER membranes. This simple lipid labelling system will be a powerful technology for real-time tracing of interorganelle lipid transport and dynamics in live cells.

\section{Results}

\section{Design, preparation and characterization of OCRs}

It is widely accepted that CPLs are synthesized de novo in the ER/Golgi and transported to other organelles through vesicular or non-vesicular traffic. ${ }^{2}$ Therefore, labelling of CPLs with a fluorescent reporter spatially specific to the ER/Golgi should be of value for analysing their intracellular trafficking by live-cell imaging. We designed an ER/Golgi-OCR, hereafter called compound 1 (Rhodol-DBCO), consisting of a fluorinated rhodol acting both as 
an ER/Golgi localizer and a fluorescent reporter, and a dibenzocyclooctyne (DBCO) as the strained alkyne (Figure 1b), on the basis of organelle-localizable protein labelling reagents we developed previously. ${ }^{12,13}$ Synthetic procedures and spectroscopic properties (absorbance, fluorescence, and $\mathrm{pH}$ dependence) of $\mathbf{1}$ are shown in the Supplementary Information and Supplementary Figure 1a, b.

The ER/Golgi specific localization of $\mathbf{1}$ was initially confirmed by confocal laser scanning microscopy (CLSM). Incubation of HeLa cells for $15 \mathrm{~min}$ at $37^{\circ} \mathrm{C}$ in culture medium containing $1(100 \mathrm{nM})$ gave strong fluorescence of 1 in the ER, Golgi, and the ER-Golgi intermediate compartment (ERGIC) (Figure 1c and Supplementary Figure 1c, d), while such fluorescence was not observed in other organelles, including mitochondria, lysosomes, peroxisomes, lipid droplets, and PMs (Supplementary Figure 1d). The intracellular concentration of 1 was estimated to be $86 \mu \mathrm{M}$ by spectroscopic analysis of the lysates prepared from cells treated with $100 \mathrm{nM} 1$ for $15 \mathrm{~min}$ (see Methods section). Given the estimated volume of the ER/Golgi (15\% of the total cell volume) ${ }^{14}$ localization of $\mathbf{1}$ in the ER/Golgi would raise the intraorganellar concentration to a final concentration of $0.57 \mathrm{mM}$, sufficient to facilitate the labelling reactions with $\mathrm{N}_{3}$-modified CPLs in the ER/Golgi. ${ }^{9}$

\section{Organelle-selective lipid labelling in live cells}

The metabolic incorporation of $\mathrm{N}_{3}$-Cho into CPLs was conducted in the conventional manner, that is, treatment of HeLa cells with $500 \mu \mathrm{M} \mathrm{N} \mathrm{N}_{3}$-Cho for $24 \mathrm{~h}^{9}{ }^{9}$ By liquid chromatography-MSMS (LC-MSMS) analysis, we confirmed that major CPLs, such as PCs and SMs, were modified with azide group with $0.3 \%$ conversion for total CPLs (Supplementary Figure $2 \mathrm{a}-\mathrm{d})$. This value is rather low compared with that in previous study by another group, ${ }^{9}$ but the low conversion may be less likely to perturb membrane dynamics and functions of the cells. For CPL labelling, HeLa cells treated with $\mathrm{N}_{3}$-Cho were subsequently incubated with 1 $(100 \mathrm{nM})$ (see Supplementary Figure 2e for optimization of probe concentration). We found that the SPAAC reaction for dye linkage completes within 15 min (Supplementary Figure 2f, g). This rapid kinetics is likely due to the effective condensation of the probe upto sub $\mathrm{mM}$ order in the organelle, which can facilitate the bimolecular reaction between the strained alkyne and azide 
group. ${ }^{12,15}$ Thereafter, the lipid fraction was extracted from the cells and analysed by thin-layer chromatography (TLC). A strong fluorescent spot was detected in the sample extracted from $\mathrm{N}_{3}$-Cho-treated cells, whereas a control sample without $\mathrm{N}_{3}$-Cho treatment only gave a faint fluorescent spot derived from decomposed products of 1 (Extended Data Fig. 1a). LC-MSMS of the main fluorescent spot successfully characterized the product from the $\mathrm{N}_{3}$-Cho/1-treated cells. As Extended Data Fig. 1b shows, several mass peaks corresponding to 1-modified PCs (1-PCs) with different fatty acid compositions were detected (Supplementary Figure $2 \mathrm{~h}-\mathrm{k}$ ), verifying that $\mathrm{N}_{3}$-PCs were labelled with 1 in live cells. Other CPLs, such as SMs, ether PCs and lyso PCs, were not detected by TLC or MS, plausibly because of their relatively low abundance in the ER/Golgi. ${ }^{2}$ These results indicate that our method selectively labels PCs.

We next sought to characterize the 1-PCs' properties. LC-MS analysis revealed that the pattern of fatty acid composition of 1-PCs was very similar to that of the natural (unlabelled) PCs (Supplementary Figure 3a). The isolated 1-PCs were susceptible to enzymatic hydrolysis by phospholipase C (PLC) and lipase, and could be incorporated into liposomes (Supplementary Figure 3b-d). Further, we confirmed that the 1-PCs were recognized and extracted from the liposome membranes by the Mmm1-Mdm12 complex, subunits of the ER-mitochondrial encounter structure (ERMES) for PC transport, ${ }^{16}$ implying that the labelled lipids could be transported via lipid transfer protein-mediated monomeric diffusion (Supplementary Figure 3e, f). Also, fluorescence recovery after photobleaching analysis successfully determined the diffusion coefficient $(D)$ of the 1-PCs in live cells to be $0.82 \pm 0.05$ $\mu \mathrm{m}^{2} / \mathrm{s}$, which is similar to the reported $D$ value for phospholipids in cellular membranes $(0.1-1.0$ $\mu \mathrm{m}^{2} / \mathrm{s}$ ) (Supplementary Figure 3g, h). ${ }^{17}$ Given these data, we conclude that the 1-PCs retain biochemical and physicochemical properties comparable with natural phospholipids.

It was found by CLSM analysis that unreacted (free) 1 was removed from the cells 15 min after washing by exchanging the culture medium (Extended Data Fig. 2a). We also confirmed that $\mathbf{1}$ does not react with any proteins (Extended Data Fig. 2b). Thus, the intracellular distribution of the 1-PCs can be fluorescently visualized with minimal background signals. As shown in Figure 1d, the fluorescence of 1-PCs was predominantly observed in the ER/Golgi in $\mathrm{N}_{3}$-Cho-treated cells, while no fluorescence derived from $\mathbf{1}$ was detected in non-treated cells. Z-stack imaging of fixed cells after labelling showed that the fluorescence of 
1-PCs colocalized well with ER/Golgi markers at any Z position (vertical direction) of the cells (Figure 1e and Extended Data Fig. 2c). Notably, such ER/Golgi-specific labelling was also observed in other types of cell, including A549 cells, HEK293T cells, and primary cultured neurons (Extended Data Fig. 2d-f), demonstrating the broad applicability and robustness of our organelle-selective lipid labelling system.

We next sought to expand this method to label mitochondrial PCs. Cationic rhodamine dyes are known to spontaneously localize into mitochondria according to the mitochondrial membrane potential. ${ }^{15,18}$ We thus employed tetraethylrhodamine as a mitochondria localizable dye and synthesized mitochondria-OCR 2 (Rhodamine-DBCO)(Figure 2a; synthesis and spectral properties are shown in Supplementary Information and Supplementary Figure 4a, b). 2 showed highly-specific mitochondrial localizability (estimated to be $\sim 60 \mu \mathrm{M}$ in mitochondria in a cell) (Supplementary Figure 4c, d). TLC analysis together with LC-MSMS of 2-PCs revealed that this probe could selectively label PCs (Extended Data Fig. 3a-c). Unreacted 2 could be removed from the cells by washing, allowing us to specifically visualize 2-PCs in mitochondria of $\mathrm{N}_{3}$-Cho-treated cells (Figure $2 \mathrm{~b}$ and Extended Data Fig. 3d-g). To visualize submitochondrial membrane structure, we performed lattice-structured illumination microscopy (SIM)-based super resolution imaging. The fast imaging rate of the lattice-SIM enabled to capture live-cell super resolution videos (Supplementary Videos 1-3) and clearly revealed that 2-PCs were located in both the outer membrane (OM) and inner membrane (IM) of mitochondria, not the mitochondrial matrix, consistent with the distribution of natural PCs in mitochondria (Figure $2 \mathrm{c}-\mathrm{f}){ }^{2}$ This observation was also supported by airy scan-based super resolution imaging with fixed-cells and electron microscopy (Extended Data Fig. 4 and 5). When the mitochondrial membrane potential was abolished by treatment of cells with carbonyl cyanide $m$-chlorophenylhydrazone $(\mathrm{CCCP})^{19}, 2$-PCs remained in mitochondria in contrast with the rapid leaking of 2 (Supplementary Figure 5). This implies that the distribution of 2-PCs is not governed by the mitochondrial membrane potential. The isolated 2-PCs were hydrolyzed by PLC and lipase, and recognized by lipid transfer protein, as well as 1-PCs (Supplementary Figure 6). Together, these data indicate that the dye modification does not bias the intramitochondrial PC behaviors, and 2-PCs can be distributed throughout the mitochondrial membrane system. 
The specific labelling of ER/Golgi and mitochondrial PCs allowed multicolor imaging of the organelle PC-lipids in live cells. As shown in Figure 2g, $\mathrm{N}_{3}$-CPLs in the ER/Golgi, mitochondria, and PMs were clearly and selectively visualized in live cells by labeling with 1, 2, and Alexa647-DBCO, respectively, thanks to the distinct fluorescence wavelength of each probe (we here concurrently employed cell-impermeable and commercially available AlexaFluor647-DBCO for labelling of PM, together with 1 and 2). These data clearly highlighted the organelle membrane-orthogonality of our method.

\section{Imaging of interorganelle translocation of labelled PCs}

We monitored the trafficking of labelled PCs from the ER/Golgi or mitochondria to other intracellular organelles by live-cell imaging. After labelling PCs with $\mathbf{1}$ in the ER/Golgi followed by washing-out the unreacted 1, cells were incubated in culture medium for $\sim 6 \mathrm{~h}$ (Protocol A, Supplementary Figure 7). As Figure 3a shows, the fluorescence signal of 1-PCs was exclusively observed in the ER/Golgi at the initial time point (just after removing unreacted 1), and the Pearson's correlation coefficient of the 1-PCs and ER/Golgi marker signals was high $(\sim 0.84)$. However, after $330 \mathrm{~min}$ the fluorescence intensity of 1-PCs in the ER/Golgi declined (Figure 3e), and, concurrently, fluorescence appeared in other organelles such as lysosomes, mitochondria, and the PM (Figure $3 b-d)$. TLC analysis confirmed that, the intracellular amount of 1-PCs did not alter in the experimental time range ( $\sim \mathrm{h})$ (Extended Data Fig. $6 \mathrm{a})$, and thus our observation is surely based on the labelled PCs itself. These results clearly indicate that the labelled PCs were transferred from the ER/Golgi to the membranes of other organelles. Given that the main pathways of lipid transport from the ER/Golgi to mitochondria and lysosome are considered to be vesicle-independent and -dependent, respectively, ${ }^{20,21}$ these observation strongly suggests that the labelled lipids can be transferred through both non-vesicular and vesicular transport systems from the ER/Golgi.

To quantitatively evaluate the efflux kinetics of PCs from the ER/Golgi, time-lapse imaging was performed (Extended Data Fig. 6b, c). The fluorescence intensity of 1-PCs in the ER/Golgi decreased during post-labelling incubation, as shown in Figure 3e; the decay curve gave a half-time of $90 \mathrm{~min}$ for efflux of PCs from the ER/Golgi. To the best of our knowledge, 
this is the first report of quantitative analysis of PC efflux from the ER/Golgi to other orgenelles, on the basis of real time imaging under live cell conditions. Colocalization analysis with organelle markers revealed that 1-PCs reached mitochondria about $1 \mathrm{~h}$ after labelling, while it took longer for translocation to the PM and lysosomes (1-2 and 4-6 h, respectively) (Figure 3bd and Extended Data Fig. 6d-f). A similar time-lapse experiment was also performed with cells where the mitochondrial PCs were labelled with 2. 2-PCs stayed in mitochondria and scarcely moved to other organelles even after $10 \mathrm{~h}$ of incubation (Extended Data Fig. 7a-c). Lysosomal translocation of 2-PCs was observed only when cells were incubated for $>20 \mathrm{~h}$. However, when the cells were stimulated by heat-shock $\left(42{ }^{\circ} \mathrm{C}\right.$ for $\left.45 \mathrm{~min}\right)$ to induce mitochondria-derived vesicles (MDVs), which are known to fuse with endosomes and lysosomes, the lysosomal transport of 2-PCs was dramatically accelerated, consistent with previous studies (Extended Data Fig. $7 \mathrm{~d}$, e). ${ }^{22}$ These results indicate that 2-PCs have potential to transfer between organelles, though they mainly remain in the mitochondria in normal conditions.

The labelling protocol of our method is quite flexible, so that the timing of OCR treatment can be varied arbitrarily. Taking advantage of this feature, we then verified the efflux rates of PCs from the ER/Golgi or mitochondria with an alternative protocol (Protocol B, Supplementary Figure 7): This protocol consists of azidocholine treatment first and then the varied incubation times prior to click chemistry with DBCO reagents so that the impact of the tagged dyes on the (transport) behavior of the labelled lipids can be disregarded. Using OCR $\mathbf{1}$, we clearly observed the incubation time-dependent decrease of $\mathrm{N}_{3}$-PCs from the ER/Golgi (Supplementary Figure $8 \mathrm{a}, \mathrm{b}$ ), which is in agreement with Figure 3e. We also performed the same experiment with OCR 2, indicating that the efflux of PCs from mitochondria is much slower than from the ER/Golgi, as shown above (Supplementary Figure 8c, d). Furthermore, by use of a cell-impearmeable DBCO dye (AFDye405-DBCO) and flow cytometry analysis, we determined the arrival time of PCs at the PM after choline metabolism to be around $1-2 \mathrm{~h}$ (Supplementary Figure 8e), which is consistent with our results obtained from the Protocol A (shown in Figure 3d). These results demonstrate that the dye tagging minimally affects the behaviors of PC dynamics.

In an attempt to gain insight into the mechanism of PC transport, we next examined whether the interorganelle lipid transport is affected by vesicle trafficking using nocodazole 
(Noc), which interferes with retrograde vesicle transport between the ER and Golgi through microtubule disruption and thereby increases Golgi-lysosome contacts, ${ }^{23,24}$ or brefeldin A (BFA), which inhibits Golgi-mediated anterograde membrane traffic. ${ }^{25}$ After labelling, cells were treated with Noc or BFA for $4 \mathrm{~h}$ and subsequently analysed by CLSM (Extended Data Fig. 8a). These drugs did not affect the efflux rate of 1-PCs from the ER/Golgi, plausibly because of the multiple trafficking pathways of PCs from the ER/Golgi to other organelles (Extended Data Fig. 8b). However, colocalization analysis of 1-PCs with organelle markers revealed that BFA strongly inhibited the translocation of 1-PCs from the ER/Golgi to lysosomes, while Noc promoted it (Extended Data Fig. 9a-c). Given the effects of BFA and Noc on the vesicle transport, it is conceivable that the Golgi-mediated pathway, rather than direct transfer from the $\mathrm{ER}$, is dominant in lysosomal lipid supply.

Together, the data make clear that our method allows fluorescence imaging of PCs and real-time tracing of their dynamic interorganellar distribution from a particular organelle membrane to others.

\section{Direct imaging of ER lipid transport to AP membranes}

Autophagosomes (APs) are vesicles induced by autophagy that are involved in the bulk degradation and recycling of intracellular components. While understanding of the physiological role of APs has greatly increased in recent decades, the origin of the AP membrane remains unclear. ${ }^{26} \mathrm{~A}$ consensus view is emerging from recent studies that the ER is the nucleation site for APs, and that contributions from other compartments (i.e. mitochondria, Golgi, and the PM) are required. ${ }^{27.28}$ However, these hypotheses have mostly been validated using protein-based fluorescent probes, not membrane lipid itself, since there were no appropriate methods capable of selectively visualizing organelle membrane lipids. As demonstrated above, our method enables us to directly label PCs in the ER/Golgi or mitochondria and to specifically trace their behaviour by live-cell imaging. We thus sought to obtain direct evidence that the AP membrane originates from the ER.

HeLa cells expressing LC3B-RFP, a canonical AP marker, were labelled with 1 and then cultured in Earle's balanced salt solution to induce AP formation (Figure 4a and Extended 
Data Fig. 10a). ${ }^{29,30}$ After a short incubation (0-30 min), a small amount of APs visualized by LC3B-RFP was observed that barely colocalized with the fluorescent signal of 1-PCs (Figure 4a and 4c). After $3 \mathrm{~h}$, the cells accumulated starvation-induced APs, whose puncta merged well with the fluorescent dots from 1-PCs (Figure 4a and 4c). Super-resolution microscopy images clearly showed that 1-PCs were detected in the AP membrane as a circle (Figure 4b). This explicitly shows that 1-PCs from the ER/Golgi were transferred to AP membranes. The number of AP vesicles including 1-PCs time-dependently increased upon starvation (Figure 4c). In sharp contrast, neither GFP with an ER retention signal sequence (KDEL-GFP) that locates in ER lumen nor ER-tracker that visualizes ATP-sensitive $\mathrm{K}^{+}$channels in the ER membrane substantially colocalized with LC3B-RFP (Figure 4c and Extended Data Fig. 10a). This indicated that these ER-resident proteins do not translocate to the starvation-induced AP membrane and are not suitable probes of membrane trafficking from the ER to APs.

Given the current membrane trafficking models, ${ }^{26}$ two possible pathways are proposed for the transport of 1-PCs from the ER/Golgi to APs: (1) lipids are directly provided to APs and/or phagophores (the membrane precursor formed upon induction of autophagy, also called isolated membrane) from the ER/Golgi; or (2) lipids are transferred from the ER/Golgi to the membrane of another organelle and then translocate to the AP membrane. To test these possibilities, we sought to catch the initial stage of AP formation in 1-treated cells by live imaging. We employed mCherry-ATG5 as a reliable phagophore marker. ${ }^{31,32}$ Like LC3B, ATG5 dots in the live cells were well colocalized with those of 1-PCs with high reproducibility, and it was estimated from 36 cells that $68 \%$ of ATG5 vesicles contain 1-PCs signal (Extended Data Fig. 10b). Most significantly, we observed that, after an ATG5 particle was generated, fluorescence of 1-PCs subsequently accumulated in the same spot on the reticular structure characteristic of the ER, and the vesicle monitored by ATG5 showed the same trajectory as that by 1-PCs (Figure 4d, e), indicating that 1-PCs are directly transferred from the ER to the AP membrane. This event was observed within $30 \mathrm{~min}$ of lipid labelling, at which time transport of 1-PCs to mitochondria, PM and lysosomes had hardly taken place. Therefore, our imaging data provide strong evidence that the ER directly supplies the membrane lipids to starvation-induced APs. ${ }^{33,34}$ 


\section{Discussion}

We here developed a new technology for organelle-selective lipid labelling under live cell conditions. Our technique is based on the metabolic incorporation of azide group into choline-phospholipids followed by a spatially limited bioorthogonal reaction, which enables in situ visualization and analysis of biosynthesized PCs in the ER/Golgi and mitochondria of living cells with minimal impacts on the biomembrane. The fast tagging rate $(\sim 15 \mathrm{~min})$ allowed for the quantitative evaluation of the efflux kinetics of PCs from the ER/Golgi and mitochondria in live-cell conditions, which has never been addressed before.

In addition, this approach allowed us to investigate the origin of autophagosome membrane by real-time tracing of organelle PCs. Although several researchers have reported that the autophagosome membrane originates from the ER, these mostly relied on indirect imaging of organelle membrane using protein-based membrane markers or electron microscopy after fixation of cells. Therefore, the origin is yet controversial. ${ }^{26,28}$ To the best of our knowledge, the present work represents the first example of direct observation of lipid molecule (not proteins) transfer from the ER to autophagosome membranes under live cell conditions in real time, which is difficult to be addressed by the conventional lipid labeling protocols lacking the organelle selectivity.

While chemical modification of the head group of PCs may affect their intrinsic properties, our results indicated that the dye-labelled PCs retain biophysical properties comparable with natural PCs and can translocate between organelles. We thus anticipate that this technology will be powerful for investigating interorganelle lipid trafficking with optical imaging and identifying key player(s) in intracellular lipid transport by combination with small molecule libraries or genome-wide knockout systems. The modular structure of OCRs will allow the extension of this strategy to other organelle membranes and the use of varied fluorescent dyes for different types of super-resolution imaging in live cells. Such future efforts should contribute to unravelling the molecular mechanisms of lipid transport, which remain largely unknown. 


\section{Acknowledgements}

We thank Eriko Kusaka (Kyoto University) for experimental support of NMR measurements. Super-resolution imaging was performed at the iCeMS Analysis Center, Institute for Integrated Cell-Material Sciences (iCeMS), Kyoto University Institute for Advanced Study (KUIAS). The electron microscopy study was supported by Keiko Okamoto-Furuta and Haruyasu Kohda (Division of Electron Microscopic Study, Center for Anatomical Studies, Graduate School of Medicine, Kyoto University). This work was funded by Grant-in-Aid for Young Scientists (18K14334) and Grant-in-Aid for Scientific Research on Innovative Areas "Integrated Bio-metal Science" (19H05764) to T.T., and the Japan Science and Technology Agency (JST) ERATO Grant No. JPMJER1802 and by a Grant-in-Aid for Scientific Research on Innovative Areas “Chemistry for Multimolecular Crowding Biosystems" (JSPS KAKENHI Grant No. 17H06348)

to I. H. We thank James Allen, DPhil, from Edanz Group (www.edanzediting.com/ac) and Mikiko Takato (Kyoto University) for editing a draft of this manuscript.

\section{Author Contributions}

T.T., I.H. and A.F. conceived the idea and directed the project. T.T., A.F., M.T. and Y.S. conducted experiments. K.N. and M.U. supervised LC-MSMS experiments and provided advice on data analysis. S.K., Y.T. and T.E. helped to conduct lipid transfer experiments. T.T. and I.H. prepared the manuscript with feedback from the other authors.

\section{Competing Financial Interests Statement}

The authors declare no competing financial interests. 


\section{References}

1. Meer, G. Van, Voelker, D. R. \& Feigenson, G. W. Membrane lipids : where they are and how they behave. Nat. Rev. Mol. Cell Biol., 9, 112-124 (2008).

2. Vance, J. E. Phospholipid synthesis and transport in mammalian cells. Traffic,16, 1-18 (2015).

3. Bumpus, T. W. \& Baskin, J. M. Greasing the wheels of lipid biology with chemical tools. Trends Biochem. Sci. 43, 970-983 (2018).

4. Maekawa, M. \& Fairn, G. D. Molecular probes to visualize the location, organization and dynamics of lipids. J. Cell Sci. 127, 4801-4812 (2014).

5. Yamaji, A. et al. Lysenin, a novel sphingomyelin-specific binding protein. J. Biol. Chem. 273, 5300-5306 (1998).

6. Kaplan, M. R. \& Simoni, R. D. Intracellular transport of phosphatidylcholine to the plasma membrane. J. Cell Biol. 101, 441-445 (1985).

7. Takakura, H. et al. Long time-lapse nanoscopy with spontaneously blinking membrane probes. Nat. Biotechnol. 35, 773-780 (2017).

8. Gupta, A., Rivera-Molina, F., Xi, Z., Toomre, D. \& Schepartz, A. Endosome motility defects revealed at super-resolution in live cells using HIDE probes. Nat. Chem. Biol. 16, 408-414 (2020).

9. Jao, C. Y., Roth, M., Welti, R. \& Salic, A. Biosynthetic labeling and two-color imaging of phospholipids in cells. ChemBioChem, 16, 472-476 (2015).

10. Neef, A. B. \& Schultz, C. Selective fluorescence labeling of lipids in living cells. Angew. Chem. Int. Ed. 48, 1498-1500 (2009).

11. Bumpus, T. W. \& Baskin, J. M. Clickable Substrate Mimics Enable Imaging of Phospholipase D Activity. ACS Cent. Sci. 3, 1070-1077 (2017).

12. Fujisawa, A., Tamura, T., Yasueda, Y., Kuwata, K. \& Hamachi, I. Chemical profiling of the endoplasmic reticulum proteome using designer labeling reagents. J. Am. Chem. Soc. 140, 17060-17070 (2018).

13. Tamura, T. \& Hamachi, I. Chemistry for covalent modification of endogenous/native proteins: From test tubes to complex biological systems. J. Am. Chem. Soc. 141, 2782-2799 (2019).

14. Alberts, B., Johnson, A., Lewis, J., Raff, M., Roberts, K., Walter, P. Molecular Biology of the Cell, 5th ed.; Garland Science: New York, 2008.

15. Yasueda, Y. et al. A set of organelle-localizable reactive molecules for mitochondrial chemical proteomics in living cells and brain tissues. J. Am. Chem. Soc. 138, 7592-7602 (2016).

16. Kawano, S. et al. Structure-function insights into direct lipid transfer between membranes by Mmm1-Mdm 12 of ERMES. J. Cell Biol. 217, 959-974 (2018).

17. Murase, K. et al. Ultrafine membrane compartments for molecular diffusion as revealed by single molecule techniques. Biophys. J. 86, 4075-4093 (2004).

18. Emaus R.K., Grunwald, R., Lemasters, J.J., Rhodamine 123 as a probe of transmembrane potential in isolated rat-liver mitochondria: spectral and metabolic properties. Biochim Biophys Acta., 850, 436-448 (1986).

19. Gao, W., Pu, Y., Luo, K. Q., Chang, D. C., Temporal relationship between cytochrome c release and mitochondrial swelling during UV-induced apoptosis in living HeLa cells. J. Cell 
Sci., 114, 2855-2862 (2001).

20. Horvath, S. E., Daum, G. Lipids of mitochondria. Prog. Lipid Res. 52, 590-614 (2013).

21. Haoxing X., Dejian, R., Lysosomal Physiology, Annu Rev Physiol., 77, 57-80 (2015).

22. Matheoud, D. et al. Parkinson's disease-related proteins PINK1 and Parkin repress mitochondrial antigen presentation. Cell, 314-327 (2016).

23. Johannes, L., Tenza, D., Antony, C. \& Goud, B. Retrograde transport of KDEL-bearing B-fragment of shiga toxin. J. Biol. Chem. 272, 19554-19561 (1997).

24. Valm, A. M. et al. Applying systems-level spectral imaging and analysis to reveal the organelle interactome. Nature. 546, 162-167 (2017).

25. Wood, S. A., Park, J. E. \& Brown, W. J. Brefeldin A causes a microtubule-mediated fusion of the trans-Golgi network and early endosomes. Cell, 67, 591-600 (1991).

26. Lamb, C. A., Yoshimori, T. \& Tooze, S. A. The autophagosome: Origins unknown, biogenesis complex. Nat. Rev. Mol. Cell Biol., 14, 759-774 (2013).

27. Hailey, D. W. et al. Mitochondria Supply Membranes for Autophagosome Biogenesis during Starvation. Cell 141, 656-667 (2010).

28. Tooze, S. A. Current views on the source of the autophagosome membrane. Essays Biochem. 55, 29-38 (2013).

29. Chan, L. L. et al. A novel image-based cytometry method for autophagy detection in living cells. Autophagy, 8, 1371-1382 (2012).

30. Munafó, D. B. \& Colombo, M. I. A novel assay to study autophagy: regulation of autophagosome vacuole size by amino acid deprivation. J. Cell Sci., 114, 3619-3629 (2001).

31. Tan, X., Thapa, N., Liao, Y., Choi, S. \& Anderson, R. A. PtdIns(4,5) $\mathrm{P}_{2}$ signaling regulates ATG14 and autophagy. Proc. Natl. Acad. Sci. 113, 10896-10901 (2016).

32. Hamasaki, M. et al. Autophagosomes form at ER-mitochondria contact sites. Nature 495, 389-393 (2013).

33. Hayashi-nishino, M., Fujita, N., Noda, T., Yamaguchi, A., Yoshimori, T. \& Yamamoto, A. A subdomain of the endoplasmic reticulum forms a cradle for autophagosome formation. Nat. Cell Biol. 11, 1433-1437 (2009).

34. Axe, E. L. et al. Autophagosome formation from membrane compartments enriched in phosphatidylinositol 3-phosphate and dynamically connected to the endoplasmic reticulum. $J$. Cell Biol. 182, 685-701 (2008). 


\section{Figure legends}

Figure 1. Endoplasmic reticulum (ER)/Golgi-selective phosphatidylcholine (PC) labelling and imaging in live cells. (a) Schematic of organelle-selective lipid labelling with organelle-localizable click reagent (OCR). (b) Molecular structure of ER/Golgi-OCR reagent 1. (c) Confocal laser scanning microscopy (CLSM) images of a HeLa cell treated with $100 \mathrm{nM} 1$ (green) for 15 min. ER-Tracker (magenta) was used for imaging both the ER and Golgi because it stains both in HeLa cells (see Supplementary Figure 1c). Scale bar, $5 \mu \mathrm{m}$. (d) ER/Golgi-specific localization of 1-PCs immediately after labelling. N $\mathrm{N}_{3}$-Cho-treated or nontreated cells were incubated with $1(100 \mathrm{nM})$ for $15 \mathrm{~min}$. After washing with fresh medium, the cells were further incubated for $30 \mathrm{~min}$, followed by CLSM analysis. Scale bar, $5 \mu \mathrm{m}$. (e) $x y$, $x z$ and $y z$ images of the labelled cells. White broken lines indicate the $x z$ and $y z$ surfaces. Scale bar, $10 \mu \mathrm{m}$. All experiments shown are representative of at least two independent measurements.

Figure 2. Mitochondria-selective PC labelling and imaging. (a) Molecular structure of mitochondrial OCR 2. (b) Mitochondria-specific localization of 2-PCs (magenta). $\mathrm{N}_{3}$-Cho-treated cells were incubated with $2(100 \mathrm{nM})$ for $15 \mathrm{~min}$. After washing with fresh medium, the cells were further incubated for $1 \mathrm{~h}$ to remove unreacted 2 and treated with Rhodamine 123 (green) for mitochondrial staining, followed by CLSM analysis. Scale bar, $5 \mu \mathrm{m}$. (c) Super resolution imaging of submitochondrial localization of 2-PCs (magenta) with Zeiss Elyra 7 with lattice SIM in a live cell. CellLight Mitochondria-GFP (Mito-GFP, green) was used to visualize the mitochondrial matrix. Scale bar, $200 \mathrm{~nm}$. (d) Enlarged image of (c). Scale bar, $200 \mathrm{~nm}$. (e, f) Graphs indicate the fluorescence intensity profiles of the 2-PCs (magenta) and Mito-GFP (green) along the white broken lines in (d). Fluorescence of 2-PCs was detected both in the outer membrane $(\mathrm{OM})$ and the inner membrane (IM) region of mitochondria. (g) Multicolor imaging of organelle membranes in HeLa cells. $\mathrm{N}_{3}$-Cho-treated cells were initially incubated with $2(100 \mathrm{nM})$ for $15 \mathrm{~min}$ at $37^{\circ} \mathrm{C}$ for labelling mitochondrial PCs. After washing the cells to remove unreacted 2 , the cells were further incubated with $1(100 \mathrm{nM})$ and AlexaFluor647-DBCO $(40 \mu \mathrm{M})$ for $1 \mathrm{~h}$ at $4{ }^{\circ} \mathrm{C}$ for labelling the ER/Golgi PCs and CPLs in the outer leaflet of PM, respectively. After washing the cells, confocal images were acquired. Scale bar, $10 \mu \mathrm{m}$. All experiments shown are representative of at least two independent measurements.

Figure 3. Live-cell tracing of interorganelle translocation of 1-PCs. (a) Confocal images of HeLa cells 0 and $330 \mathrm{~min}$ after lipid labelling with 1. Colocalization was quantified using Pearson's correlation coefficient (PCC) for 1-PCs and the signal from ER/Golgi markers. In this experiment, the fluorescence intensities of 1-PCs were adjusted to be the same in both images. Scale bar, $5 \mu \mathrm{m}$. (b, c, d) Colocalization analysis of 1-PCs with (b) lysosomes, (c) mitochondria, and (d) plasma membrane (PM). Yellow arrowheads indicate 1-PCs were transferred from ER/Golgi to the other organelle at the indicated time. Scale bar, $2 \mu \mathrm{m}$ for (b, d), $0.5 \mu \mathrm{m}$ for (c). (e) Efflux curve of 1-PCs from the ER/Golgi. The fluorescence intensity of 1-PCs observed in the ER/Golgi (stained with ER-Tracker) was quantified. Scatter plots show mean \pm S.D. $(n=13$, $12,13,12,12$, and 11 cells for data points left to right). Inset, representative images of the time-lapse imaging. Scale bar, $5 \mu \mathrm{m}$. All experiments shown are representative of at least two 
independent measurements.

Figure 4. Transport of 1-PCs from the ER to autophagosome (AP) membranes. (a) Time-lapse images of starved HeLa cells. White arrowheads indicate 1-PCs (green) colocalized with LC3B-RFP (an AP marker, magenta). Scale bar, $5 \mu \mathrm{m}$. (b) Colocalization of 1-PCs with LC3B-RFP in AP membranes. Graphs indicate the fluorescence intensity profiles of the 1-PCs (green) and LC3B-RFP (magenta) along the white broken lines in the images. Scale bar, $1 \mu \mathrm{m}$. (c) Quantification of AP vesicles overlapping with ER/Golgi or mitochondrial probes (1-PCs, KDEL-GFP, and ER-Tracker) during starvation. The number of AP vesicles including the fluorescence signals of these markers was counted. This number was divided by the total number of APs to determine the percentage of APs that overlapped with the marker in each cell. Bar graphs represent mean $\pm \mathrm{SD}(n=10$ cells). (d) Time-lapse images of AP formation in 1-treated cells. HeLa cells transfected with mCherry-ATG5 (a phagophore marker) were labelled with 1. After washing, the cells were incubated in Earle's balanced salt solution for $30 \mathrm{~min}$, followed by CLSM analysis. Scale bar, $1 \mu \mathrm{m}$. (e) Trajectories of the ATG-5 vesicle (magenta) and the 1-PCs vesicle (green) shown in (d). All experiments shown are representative of at least two independent measurements.

\section{Online Methods}

\section{Synthesis}

$\mathrm{N}_{3}$-Cho was prepared as previously described. ${ }^{9}$ Synthesis of reagents $\mathbf{1}$ and $\mathbf{2}$ are described in Supplementary Information.

\section{General materials and methods for the biochemical/biological experiments.}

Unless otherwise noted, all chemical reagents and proteins/enzymes were purchased from commercial suppliers (Thermo Fisher Scientific, TCI, Sigma-Aldrich, Bio-Rad, FUJIFILM, Nacalai tesque, Avanti Polar Lipids) and used without further purification. UV-vis absorption spectra were measured on a Shimazu UV-2600 spectrophotometer. Excitation/Emission spectra were measured on a Perkin Elmer LS-55 Luminescence Spectrometer. Cell imaging was performed with a confocal laser scanning microscope (CLSM) (Carl Zeiss LSM800, LSM800 Airyscan). SDS-polyacrylamide gel electrophoresis (SDS-PAGE) was carried out using a Bio-Rad Mini-Protean III electrophoresis apparatus. Fluorescence gel and TLC images were acquired with an imagequant LAS4000 (GE Healthcare) or FUSION (Vilber-Lourmat).

\section{Cell culture for imaging}

HeLa, A549 and HEK293T cells were cultured on $35 \mathrm{~mm}$ glass-bottom multi well dish (MATSUNAMI) in Dulbecco's Modified Eagle's Medium (DMEM, Sigma-Aldrich) supplemented with 1\% Antibiotic-Antimycotic (Anti-Anti, Gibco) and 10\% fetal bovine serum (FBS, Sigma) under a humidified atmosphere of $5 \% \mathrm{CO}_{2}$ in air at $37{ }^{\circ} \mathrm{C}$. Rat primary hippocampal neurons were prepared from 16-day-old Sprague Dawley rat embryos (unknown 
gender) as previously described. ${ }^{35}$ The neurons were cultured on poly-D-lysine-coated $35 \mathrm{~mm}$ glass-bottom dish (ibidi) in Neurobasal plus medium supplemented with 2\% B27 supplement (Thermo Fisher Scientific) and $1 \%$ penicillin streptomycin (Gibco) under a humidified atmosphere of $5 \% \mathrm{CO}_{2}$ in air at $37{ }^{\circ} \mathrm{C}$. All experimental procedures were performed in accordance with the National Institute of Health Guide for the Care and Use of Laboratory Animals and approved by the Institutional Animal Use Committees of Kyoto University.

\section{Metabolic incorporation of $\mathrm{N}_{3}$-Cho into CPLs and labelling of the $\mathrm{N}_{\mathbf{3}}$-CPLs with OCRs}

Cells were incubated in the culture medium containing $\mathrm{N}_{3}$-Cho $(500 \mu \mathrm{M})$ for $24 \mathrm{~h}$. The cells were washed twice with serum-free DMEM (no Phenol Red, $25 \mathrm{mM}$ HEPES, Gibco) and incubated in serum-free DMEM containing OCRs $(100 \mathrm{nM})$ for $15 \mathrm{~min}$. To remove free OCRs inside cells, the cell culture medium was replaced to fresh DMEM supplemented with Anti-Anti and $10 \% \mathrm{FBS}$, and further incubated for $30 \mathrm{~min}$ or $1 \mathrm{~h}$ for $\mathbf{1}$ or $\mathbf{2}$, respectively.

\section{Cell imaging}

Cell imaging was performed using a Carl Zeiss LSM800 or a LSM880 equipped with Airyscan super-resolution system and a $\times 100$ oil immersion objective lens, a Carl Zeiss Elyra 7 with lattice SIM with a $\times 100$ oil immersion objective lens, or Leica TCS SP8 equipped with a $\times 63$ oil immersion objective lens. The obtained images were analysed by ZEN Viewer software or by LAS X SP8 Dye Finder. For live-cell imaging, cells were maintained on a microscope at $37{ }^{\circ} \mathrm{C}$ with a continuous supply of $5 \% \mathrm{CO}_{2}$ in air by using a stage-top incubator (Tokai Hit). For heat-shock experiment, cells were heated at $42{ }^{\circ} \mathrm{C}$ in the incubator. Organelles were stained by the following organelle markers, according to manufactures' instruction: ER-Tracker Green, ER-Tracker Red, Mito-Tracker Deep Red, Lyso-Tracker Red DND-99, Lyso-Tracker Deep Red, CellLight Golgi-GFP, CellLight Golgi-RFP, CellLight Peroxisome-GFP, Premo Autophagy Sensor LC3B-GFP, Premo Autophagy Sensor LC3B-RFP, CellLight ER-GFP (KDEL-GFP), CellLight ER-RFP (KDEL-RFP), and CellLight Mitochondria-GFP obtained from Thermo Fisher Scientific; Rhodamine123 and Mito-Red obtained from Dojindo. Cells were transfected with EGFP-F ${ }^{36}$, mCherry-F ${ }^{36}$, ERGIC53-DsRed ${ }^{37}$, mCherry-TOMM20-N-10 (gifted from Dr. M. Davidson, Addgene, 55146), mEmerald-TOMM20-N-10 (gifted from Dr. M. Davidson, Addgene, 54282), DsRed2-Peroxi (Clontech), or mCherry-ATG5 (gifted from Dr. M. Davidson, Addgene, 55146) using lipofectamine 2000 (Invitrogen) for staining plasma membrane, ERGIC, mitochondrial outer membrane, peroxisome, or phagophore. Lipid droplets were labeled with BODIPY $665 / 676$ as previously described. ${ }^{24}$ The fluorescence images were obtained by excitation at $473 \mathrm{~nm}, 559 \mathrm{~nm}$ and $635 \mathrm{~nm}$. Co-localization was quantified using Pearson's correlation coefficient with ZEN Viewer software or by LAS X SP8 Dye Finder. For fixation analysis, cells were washed with phosphate-buffered saline (PBS (-)), and fixed with 4\% paraformaldehyde (PFA) phosphate buffer solution (FUJIFILM) at r.t. for $15 \mathrm{~min}$. After washing with PBS (-) once, the sample was analysed by CLSM.

\section{Determination of intracellular concentration of OCRs.}

HeLa cells $\left(1.0 \times 10^{7}\right.$ cells $)$ were incubated in serum-free DMEM containing OCRs $(100 \mathrm{nM})$ for $15 \mathrm{~min}$. The cells were washed twice with PBS (-) and harvested using trypsin solution (Gibco). The cell number was counted by CountessII FL (Thermo Fisher Scientific), and the cells were lysed by sonication in $500 \mu \mathrm{L}$ of $1 \%$ SDS lysis buffer $(25 \mathrm{mM}$ Tris-HCl, $150 \mathrm{mM} \mathrm{NaCl}, 1 \%$ 
Nonidet P-40, 1\% SDS, $1 \%$ sodium deoxycholate, $\mathrm{pH}$ 7.6). The lysate was centrifuged at 16,000 $\mathrm{g}$ for $3 \mathrm{~min}$, and the supernatant was collected. The concentration of OCRs in the lysate was determined by fluorescent intensity measured with a LS55 fluorescent spectrometer (PerkinElmer) using a standard dye solution of known concentration. Finally, the averaged intracellular concentration of OCRs in a single cell was determined from the cell number, sample volume and a typical single cell volume (HeLa cell volume was reported as $2.4 \mathrm{pL}$ ) ${ }^{38}$.

\section{Lipid extraction and TLC analysis.}

After labelling, the cells $\left(1.0 \times 10^{6}\right.$ cells $)$ were washed twice with PBS (-) and added $2 \mathrm{~mL}$ of ice-cold methanol containing $10 \mathrm{mM}$ azidoacetic acid (Sigma-Aldrich) as a quencher of SPAAC reaction. Lipids were extracted from the homogenate by a Bligh-Dyer method. ${ }^{39}$ The extracted lipids was dissolved in $50 \mu \mathrm{L}$ of chloroform/methanol $=1 / 1$ solution, spotted on thin layer chromatography (Wako, silica gel 70), and developed with chloroform-methanol-28\% ammonia $(14: 5: 1, \mathrm{v} / \mathrm{v})$. The fluorescence signal of labelled lipids was detected by an imagequant LAS4000 (GE Healthcare) or Fusion (Vilber-Lourmat). Lipid species were stained by CAM stain (10 v/v\% concentrated sulfuric acid, $\left.1 \mathrm{w} / \mathrm{v} \% \mathrm{Ce}\left(\mathrm{SO}_{4}\right)_{2}, 5 \mathrm{w} / \mathrm{v} \%\left(\mathrm{NH}_{4}\right)_{6} \mathrm{Mo}_{7} \mathrm{O}_{24} \cdot 4 \mathrm{H}_{2} \mathrm{O}\right)$.

\section{LC/MS analysis}

Whole lipids from $\mathrm{N}_{3}$-Cho-treated cells were extracted by Bligh-Dyer method. The labelled products were isolated from TLC by scratching it and extracted with methanol. These samples were subjected to LC-MSMS analysis using a HPLC system LC-30AD (Shimadzu) coupled to a triple quadrupole mass spectrometer LCMS-8040 (Shimadzu) equipped with an electrospray source. The extracted lipids were dissolved in $200 \mu \mathrm{L}$ of MS-grade methanol (FUJIFILM), filtered and injected $5 \mu \mathrm{L}$ into a Kinetex reverse phase C8 HPLC column (Phenomenex, $2.6 \mu \mathrm{m}$, $2.1 \times 150 \mathrm{~mm}$ ). The mobile phases consisted of (A) $10 \mathrm{mM}$ ammonium formate and (B) 2-propanol/acetonitrile/10mM ammonium formate $(45 / 45 / 10, \mathrm{v} / \mathrm{v} / \mathrm{v})$. The pump controlling the gradient of mobile phase B was programmed as follows: an initial isocratic flow at $20 \% \mathrm{~B}$ for 1 min, a linear increase to $40 \% \mathrm{~B}$ for $1 \mathrm{~min}$, an increase to $92.5 \% \mathrm{~B}$ using a curved gradient for 18 min, followed by a linear increase to $100 \% \mathrm{~B}$ for $2 \mathrm{~min}$, and a hold at $100 \% \mathrm{~B}$ for $8 \mathrm{~min}$. The total flow rate was $0.3 \mathrm{ml} / \mathrm{min}$, the column temperature was $45^{\circ} \mathrm{C}$, collision energy $-50 \mathrm{~V}(\mathrm{Q} 1$ : unit, Q3 : low). For the extract from $\mathrm{N}_{3}$-Cho-treated cells, fragment ion $\mathrm{m} / \mathrm{z}$ was set to be a 184.07 (native choline) and $239.09\left(\mathrm{~N}_{3}\right.$-choline). Total ion current chromatogram of choline- and $\mathrm{N}_{3}$-Cho-containing lipids was recorded to estimate the incorporation efficiency of $\mathrm{N}_{3}$-Cho. For the detection of 1-PCs, fragment ion $\mathrm{m} / \mathrm{z}$ was set to be 782.3. Precursor ion 1452.8, 1498.8, and $1526.0 \mathrm{~m} / \mathrm{z}$ were used to characterize their fatty acid composition by MSMS analysis using negative fragment ion scan mode. In the case of 2-PCs, fragment ion $m / z$ was set to be 912.49 .

\section{Treatment of labelled cells with Noc and BFA.}

After labelling with 1, the cells were incubated in DMEM (with 10\% FBS) containing DMSO (control), Noc $(1 \mu \mathrm{M})$ or BFA $(20 \mu \mathrm{M})$ for $4 \mathrm{~h}$. After washing with serum-free DMEM, the cells were incubated in serum-free DMEM containing ER-Tracker Red $(1 \mu \mathrm{M})$ and Lyso-Tracker Deep Red (50 nM) for 30 min, followed by CLSM analysis.

\section{Induction of autophagy by serum-starvation.}


After labelling with chemical probes (1, 2, or ER-Tracker) or transfected with CellLight ER-GFP (KDEL-GFP), the cells were incubated in Earle's Balanced Salt Solution (Thermo Fisher Scientific). The number of LC3B-positive AP vesicles including the fluorescence signals of 1-PCs, 2-PCs, ER-Tracker Green or KDEL-GFP was counted. This number was divided by the total number of APs to determine percent of APs that overlapped with the marker in each cell.

\section{Preparation of GUVs containing labelled lipids}

Giant unilamellar vesicles (GUVs) were made from hybrid films of lipids and agarose according to the previous report. ${ }^{40,41}$ Briefly, ternary mixtures of DOPC (1,2-di-oleoyl-sn-glycero-3-phosphocholine)/1-PCs/RhodamineB(Rhod)-DOPE

(1,2-dioleoyl-sn-glycero-3-phosphoethanolamine-n-(lissamine rhodamine B sulfonyl)) (30 nmol, $0.05 \mathrm{nmol}$, $0.1 \mathrm{nmol}$, respectively) or DOPC/2-PCs/NBD-DOPE (1,2-dioleoyl-sn-glycero-3-phosphoethanolamine-N-(7-nitro-2-1,3-benzoxadiazol-4-yl)) nmol, $0.023 \mathrm{nmol}, 0.15 \mathrm{nmol}$, respectively) were dissolved in $30 \mu \mathrm{L}$ of chloroform. The lipid mixtures were deposited onto agarose (Type-IX-A, Sigma-Aldrich) film and dried under vacuum. The lipid on agarose film was immersed in PBS containing $100 \mathrm{mM}$ sucrose, $0.1 \mathrm{mM} \mathrm{CaCl}_{2}$ and $1 \mathrm{mM} \mathrm{MgCl} 2$. The supernatant was dropped into PBS containing $100 \mathrm{mM}$ glucose, $0.1 \mathrm{mM}$ $\mathrm{CaCl}_{2}$ and $1 \mathrm{mM} \mathrm{MgCl} 2$ in gelatin-coated glass-bottom dishes. The resultant GUVs were observed using an epifluorescence microscope (IX83, OLYMPUS) with a 60x objective lens.

\section{Lipid transfer assay}

To monitor lipid extraction from liposomes, $10 \mu \mathrm{M}$ Mmm1-Mdm12 complex ${ }^{16}$ was mixed with $200 \mu \mathrm{M}$ donor liposome, whose lipid composition is POPC (Avanti)/the isolated 1-PCs or 2-PCs $=80 / 20$, in $150 \mu \mathrm{L}$ TBS $(20 \mathrm{mM}$ Tris- $\mathrm{HCl}, 150 \mathrm{mM} \mathrm{NaCl}, \mathrm{pH} 7.5)$ and incubated at $37{ }^{\circ} \mathrm{C}$ for 30 min. After incubation, the reaction mixtures were put into $5 \mathrm{~mL}$ of an ultracentrifuge tube and mixed with $300 \mu \mathrm{L}$ TBS containing 60\% (wt/vol) sucrose. The mixture was subsequently overlaid by $2 \mathrm{~mL}$ TBS containing 30\% (wt/vol) sucrose, $2 \mathrm{~mL}$ TBS containing 10\% (wt/vol) sucrose, and then $550 \mu \mathrm{L}$ TBS without sucrose. After ultracentrifugation at $200,000 \mathrm{~g}$ for $1.5 \mathrm{~h}$, protein-containing fractions were analyzed by fluorescence excited at $480 \mathrm{~nm}$ and observed at $534 \mathrm{~nm}$ for 1-PCs or at $560 \mathrm{~nm}$ and observed at $570 \mathrm{~nm}$ for 2-PCs, with a FP-6500 spectrofluorometer (JASCO).

\section{FRAP analysis}

HeLa cells were labelled with 1 as described in Methods section. Bleaching was performed with a circular spot (diameter : $3.16 \mu \mathrm{m}$ ) using $488 \mathrm{~nm}$ laser operating at $100 \%$ laser power for 30 times with a Carl Zeiss LSM800. Fluorescence recovery was monitored at $5 \mathrm{sec}$ intervals. The diffusion coefficient $(D)$ was calculated by fitting the raw data to a single exponential association equation and a two-dimensional diffusion equation described below. ${ }^{42}$

$$
\begin{aligned}
& F_{\mathrm{t}}=F_{0}+\left(F_{\max }-F_{0}\right)\left(1-e^{-k t}\right) \\
& t_{1 / 2}=\ln 2 / k \\
& D=\left(r^{2} \mathrm{~g}\right) /\left(4 t_{1 / 2}\right)
\end{aligned}
$$


$F_{t}$ is the corrected fluorescence recorded from region of interest (ROI) at time $t . F_{0}$ and $F_{\max }$ are the fluorescence values obtained by extrapolation to immediately after the bleach and at infinite time after recovery, respectively. $t_{1 / 2}$ is the half-time for recovery. $k$ is the first-order rate constant for recovery. $r$ is the radius of the beam. $g$ is a correction factor for bleaching. All results are expressed as means \pm S.D.

\section{Evaluation of nonspecific reaction to proteins with OCRs}

HeLa cells $\left(5.0 \times 10^{6}\right.$ cells $)$ were cultured in DMEM on $35 \mathrm{~mm}$ dishes under a humidified atmosphere of $5 \% \mathrm{CO}_{2}$ in air at $37{ }^{\circ} \mathrm{C}$ for $48 \mathrm{~h}$. After $\mathrm{N}_{3}$-Cho incorporation, the cells were incubated in serum free DMEM containing OCRs $(100 \mathrm{nM})$ for $15 \mathrm{~min}$ or control compounds (ERM $6^{12}$ and MRM1a ${ }^{15}, 10 \mu \mathrm{M}$ ) for $3 \mathrm{~h}$. The cells were washed twice with chilled PBS and lysed by sonication in $200 \mu \mathrm{L}$ of RIPA buffer ( $25 \mathrm{mM}$ Tris, $150 \mathrm{mM} \mathrm{NaCl}, 1 \%$ Nonidet P-40, $4 \%$ Sodium dodecyl sulfate, $1 \%$ sodium deoxycholate, $\mathrm{pH} 7.6$ ) containing $1 \%$ protease inhibitor cocktail set III (Novagen) at $4{ }^{\circ} \mathrm{C}$. Concentration was measured with BCA assay kit (Thermo Fisher Scientific) and the solution was boiled in $2 \times$ SDS-PAGE loading buffer (125 mM Tris, $10 \%$ glycerol, $0.01 \%$ bromophenol blue, $0.1 \%$ SDS, pH 6.8) containing $200 \mathrm{mM}$ DTT for 5 min. The samples were resolved by SDS-PAGE, and the labelled proteins were visualized by LAS4000 fluorescence gel imager.

\section{Hydrolysis of labelled PCs by lipase}

The isolated 1-PCs or 2-PCs $(20 \mathrm{pmol})$ were dissolved in $10 \mu \mathrm{L}$ of EtOH. To this solution, a 90 $\mu \mathrm{L}$ of $0.1 \mathrm{M} \mathrm{KH}_{2} \mathrm{PO}_{4}$ buffer ( $\mathrm{pH} 7.0$ ) containing $90 \mu \mathrm{g}$ of lipase (Wako, \#122-02651) was added. The mixture was incubated at $37^{\circ} \mathrm{C}$ for $24 \mathrm{~h}$. The reaction mixture was mixed with $10 \mathrm{uL}$ of $1: 1$ $\mathrm{CHCl}_{3} / \mathrm{MeOH}$ solution and spotted on TLC. The TLC was developed with chloroform-methanol-28\% ammonia (14:5:1, v/v).

\section{Hydrolysis of labelled PCs by PLC}

The isolated 1-PCs or 2-PCs (80 pmol) were dissolved in $10 \mu \mathrm{L}$ of EtOH. To this solution, add $30 \mu \mathrm{L}$ of a reaction buffer $\left(25 \mathrm{mM}\right.$ Tris-HCl, $\left.3.2 \mathrm{mM} \mathrm{CaCl}_{2}, \mathrm{pH} 7.3\right)$ containing $10 \mu \mathrm{g}$ of PLC (Sigma, P4039) was added. The mixture was incubated at $37^{\circ} \mathrm{C}$ for $1 \mathrm{~h}$. The reaction mixture was mixed with $10 \mathrm{uL}$ of $1: 1 \mathrm{CHCl}_{3} / \mathrm{MeOH}$ solution and spotted on TLC. The TLC was developed with chloroform-methanol-28\% ammonia (14:5:1, v/v).

\section{Electron microscopy}

After mitochondrial PC labelling with 2, the cells were fixed with 4\% paraformaldehyde/1\% glutaraldehyde mixture in $0.1 \mathrm{M}$ sodium phosphate $(\mathrm{pH} 7.4)$ for $30 \mathrm{~min}$ at room temperature, followed by overnight at $4{ }^{\circ} \mathrm{C}$. The samples were post-fixed with $1 \%$ osmiumtetraoxide in $0.1 \mathrm{M}$ phosphate buffer, dehydrated with a graded series of ethanol, and embedded in epoxy-resin Luveak (NacalaiTesque). Ultra-thin sections were prepared on an ultramicrotome (EM UC6 ; Leica, Heiderberg, Germany) and mounted on 200-mesh nickel grids coated with collodion (Nisshin EM Co., Ltd., Tokyo, Japan). The grids were incubated in $20 \%$ normal goat serum (NGS) in PBS for $10 \mathrm{~min}$ at room temperature. After washing with PBS, the grids were stained with anti-tetraethylrhodamine antibody ${ }^{15}$ (x250 dilution with $2 \%$ NGS), followed by anti-rabbit IgG $10 \mathrm{~nm}$ gold preadsorbed (abcam, ab27234, x100 dilution with 2\% NGS). After washing with PBS and $0.1 \mathrm{M}$ phosphate buffer, the samples were fixed with $1 \%$ glutaraldehyde in $0.1 \mathrm{M}$ 
sodium phosphate, rinsed in distilled water, counterstained with uranyl acetate and lead citrate for $30 \mathrm{~min}$, and observed under an electron microscope (H-7650, Hitachi Tokyo, Japan).

\section{Flow cytometry}

K562 cells were cultured on non-treated cell culture dishes (IWAKI) in choline-free RPMI-1640 (Wako) supplemented with 1\% Penicillin-Streptomycin (Wako) and 10\% fetal bovine serum (FBS, Sigma) under a humidified atmosphere of $5 \% \mathrm{CO}_{2}$ in air at $37{ }^{\circ} \mathrm{C}$ for $18 \mathrm{~h}$. The cells $(3 \times$ $10^{4}$ cells) were incubated in the culture medium containing $\mathrm{N}_{3}$-Cho $(500 \mu \mathrm{M})$ for $0-12 \mathrm{~h}$, then washed with the medium containing 4\% FBS and further incubated at $15{ }^{\circ} \mathrm{C}$ for $15 \mathrm{~min}$. The cells were subsequently incubated in the medium containing AFDye 405-DBCO (Fluoroprobes, $100 \mu \mathrm{M}$ ) at $15{ }^{\circ} \mathrm{C}$ for $30 \mathrm{~min}$, washed with ice-cold medium containing $10 \% \mathrm{FBS}$, and analyzed by a flow cytometer (SONY, MA900).

\section{Data availability Statement}

The authors declare that all data supporting the findings of this study are available within the paper and the Supplementary Information files. The materials and data reported in this study are available upon reasonable request from the corresponding author.

\section{Methods-only References}

35. Wakayama, S. et al. Chemical labelling for visualizing native AMPA receptors in live neurons. Nat. Commun. 8, 14850 (2017).

36. Kiyonaka, S. et al. Allosteric activation of membrane-bound glutamate receptors using coordination chemistry within living cells. Nat. Chem. 8, 958-967 (2016).

37. Miki, T. et al. A conditional proteomics approach to identify proteins involved in zinc homeostasis. Nat. Methods 13, 931-937 (2016).

38. Yoshii, T., Mizusawa, K., Takaoka, Y. \& Hamachi, I. Intracellular protein-responsive supramolecules: protein sensing and in-cell construction of inhibitor assay system. $J . A m$. Chem. Soc. 136, 16635-42 (2014).

39. Bligh, E. G. \& Dyer, W. J. A rapid method of total lipid extraction and purification, Can. J. Biochem. Physiol., 37, 911-917 (1959).

40. Horger, K. S., Estes, D. J., Capone, R. \& Mayer, M. Films of agarose enable rapid formation of giant liposomes in solutions of physiologic ionic strength. J. Am. Chem. Soc. 131, 18101819 (2009).

41. Ikenouchi, J. et al. Lipid polarity is maintained in absence of tight junctions. J. Biol. Chem. 287, 9525-9533 (2012).

42. Pantazaka, E. \& Taylor, C. W. Differential distribution, clustering, and lateral diffusion of subtypes of the inositol 1,4,5-trisphosphate receptor. J. Biol. Chem. 286, 23378-23387 (2011). 